\title{
Modificaciones en la grasa del atún blanco (Thunnus alalunga) debidas a la fabricación y almacenamiento de su conserva
}

\author{
Por M. ${ }^{\text {a }}$ T. García Arias, A. M. a Castrillón y M. ${ }^{\text {a }}$ P. Navarro* \\ Instituto de Nutrición y Bromatología \\ Facultad de Farmacia - Ciudad Universitaria - 28040 Madrid
}

\section{RESUMEN}

Modificaciones en la grasa del atún blanco (Thunnus alalunga) debidas a la fabricación y almacenamiento de su conserva.

Se estudiaron los cambios que se producen en la grasa del atún blanco en cada una de las etapas que intervienen en el proceso de fabricación y almacenamiento de su conserva: cocción en salmuera y esterilización practicada en aceite de soja. Se estudiaron dos tiempos diferentes de esterilización (55 y 90 minutos) y el almacenamiento por espacio de 1 año.

La cocción en salmuera no afectó a la composición en ácidos grasos de los lípidos del pescado ni a la relación $n-3 / n-6$. Sin embargo, durante la esterilización, el atún absorbió el aceite utilizado como cobertura, lo que produjo un aumento porcentual en el nivel de C18:1, C18:2, C18:3 y una disminución en C20:5 y C22:6. La relación ácidos grasos insaturados a saturados (PS/S) cambió y la n-3/n-6 disminuyó desde 7,03 a 0,59 comparado con atún crudo. En el pescado en conserva, debido al enriquecimiento total en grasa, se observaron estos cambios pero más atenuada la pérdida de los poliinsaturados propios, así como la clara ganancia de C18:1, C18:2 y C18:3, que se acentuó aún más tras el año de almacenamiento.

Se discute la conveniencia de utilizar para la conserva de atún blanco un aceite menos rico en poliinsaturados que mantenga mejor la relación $n-3 / n-6$ originaria del pescado fresco.

PALABRAS-CLAVE: Aceite de cobertura - Acido graso (composición en) - Almacenamiento - Conserva - Grasa (modificación) - Pescado.

\section{SUMMARY}

Changes in white tuna (Thunnus alalunga) fat due to cannig process and storage preserve.

Modifications in fatty acid composition in white tuna (Thunnus alalunga) ocurring at each stage of the cannig process, boiling and sterilization in soybean oil, were studied. Boiling was performed in brine and two sterilization times (55 and 90 minutes) as well as the effects of storage for one year were tested. Boiling had no effect on fatty acid composition or the $n-3 / n-6$ ratio. However canned tuna absorbed soybean oil during sterilization, causing an increase of the percentage of $\mathrm{C} 18: 1, \mathrm{C} 18: 2$ and $\mathrm{C} 18: 3$ and a decrease in $\mathrm{C} 20: 5$ and C22:6. Therefore, unsaturated fatty acids to saturated fatty acids (PS/ S) ratio changed and the $n-3 / n-6$ ratio declined from 7.03 to 0.59 compared with raw tuna. Due to the whole fat enrichment, canned fish showed a slight loss of PUFA (C20:5 and C22:6) and a increase of $C_{18: 1}, C_{18: 2}$ and $C_{18: 3}$. The latter effect was more marked in the preserves as a consequence of one year storage. The convenience of using for cannig an oil containing a lower amount of polyunsaturated fatty acid in order to remain the original $n-3 / n-6$ ratio of the raw tuna is discussed.
KEY-WORDS: Coating oil - Fat (modification) - Fatty acid (composition in) - Fish - Preserve - Storage.

\section{1.-INTRODUCCIÓN}

Por la calidad de su proteína y por el contenido de vitaminas y minerales el pescado se presenta como un alimento de gran calidad. Pero actualmente, desde el punto de vista nutritivo y sanitario en general, el consumo de las especies azules se estimula en base preferentemente a las características de su grasa, rica en ácidos grasos poliinsaturados (PUFA) de la familia $n-3$ especialmente. Su papel beneficioso, en relación con las enfermedades cardiovasculares, reside en que los n-3 poseen una capacidad superior a los de cualquier droga conocida para inducir descenso en los triglicéridos plasmáticos (1), además del efecto hipocolesterolémico que ejercen los n-6 (2).

El atún blanco o bonito del norte es una especie grasa con alto contenido en $n-3, y$, al igual que el salmón, muy rica en C22:6. Además, en nuestro país, goza de enorme interés económico, así el valor de mercado de sus capturas (según datos del MAPA para 1987) fue alrededor del $40 \%$ del total de las de los pescados azules. Más del $65 \%$ del bonito desembarcado se destina a la elaboración de conservas, que en parte son comercializadas interiormente ya que el atún es el pescado más consumido en forma de conserva por nuestra población: $2.24 \mathrm{~g}$ por persona y día (3), y en gran medida son exportados.

No obstante, casi toda la información disponible acerca de las características de la grasa de este y de otros pescados se refiere a las especies crudas (4) y pocos datos existen respecto a los cambios que pueden producirse durante los procesos industriales o domésticos en los ácidos grasos del pescado (5). En esta línea, aunque escasea la bibliografía, se sabe que durante el enlatado de sardinas tiene lugar un cambio en la naturaleza de su grasa (6), y en otro proceso que también implica calentamiento en un 
medio con aceite, la fritura de pescado, se produce un intercambio entre la grasa, el agua del alimento y el aceite empleado (7) (8) (9).

Por todo ello nos pareció importante estudiar los cambios que se producen en la grasa del atún blanco durante el proceso de fabricación y almacenamiento de su conserva. Considerando, además, dos variables de tiempo de esterilización, uno más largo, habitual en la industria, y otro más corto pero suficiente desde el punto de vista microbiológico.

\section{2.-MATERIAL Y METODOS}

\subsection{Preparación de la muestra.}

Para el estudio se utilizaron cuatro ejemplares de atún blanco (Thunnus alalunga) entre $4.6 \mathrm{Kg}$ y $5.2 \mathrm{Kg}$ de peso, capturados por un barco comercial en junio de 1986, que fueron refrigerados en el barco, congelados y almacenados $\mathrm{a}-18^{\circ} \mathrm{C}$ durante 3 meses hasta el momento de la fabricación de la conserva. Las

\section{Esquema 1}

Proceso de fabricación de la conserva de atún blanco (Thunnus alalunga)

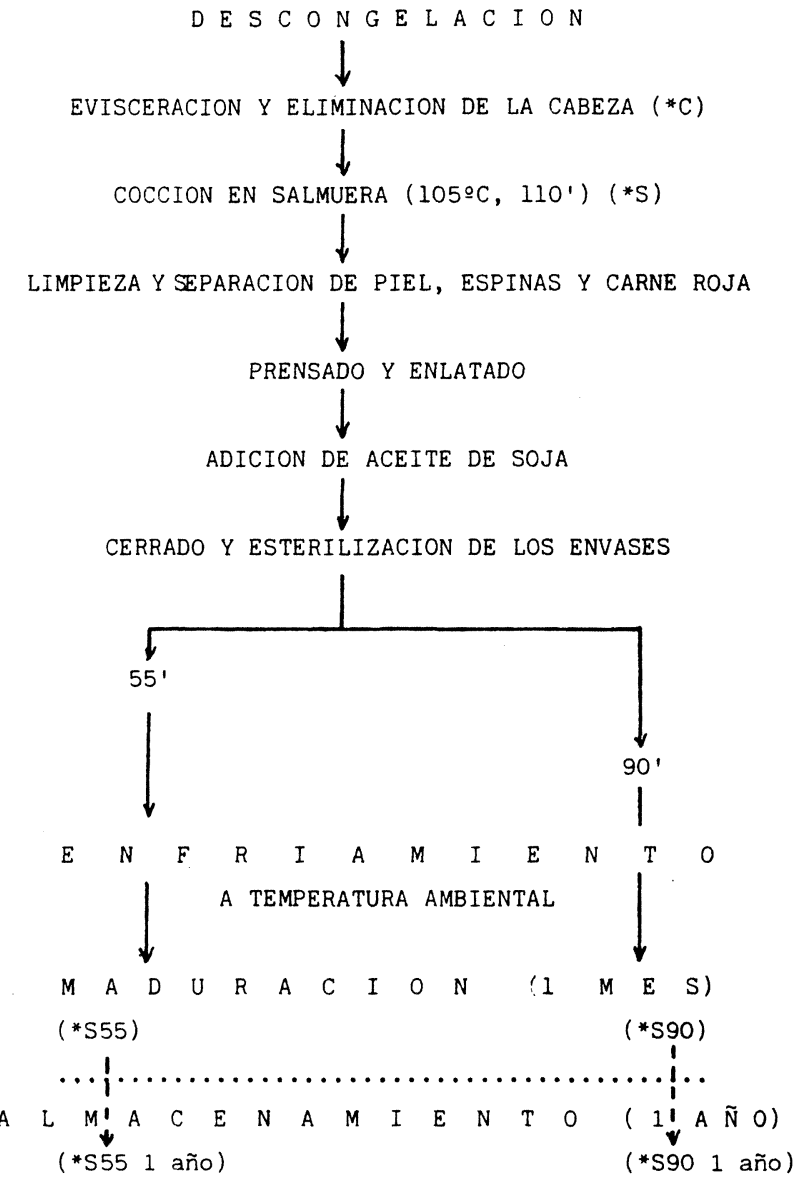

distintas etapas de la misma se realizaron en el Instituto de Investigaciones marinas de Vigo del C.S.I.C., siguiendo el Esquema I, en el cual se señalan entre paréntesis los puntos donde se hicieron las distintas tomas de muestras.

El atún descongelado, primeramente se descabezó, evisceró y lavó $\left({ }^{\star} \mathrm{C}\right)$ y después se coció en salmuera $\left(130 \mathrm{~g}\right.$ de sal/litro) a $105^{\circ} \mathrm{C}$ durante 110 minutos ( $\left.{ }^{\star} S\right)$. A continuación se limpió, separando piel, espinas y carne roja, y se procedió al enlatado de los lomos en envases de forma OL-120 con adición de aceite de soja. Las cantidades incluidas en cada envase fueron $80 \mathrm{~g}$ de pescado y $35 \mathrm{cc}$ de aceite, proporciones comúnmente utilizadas en la industria de este pescado para este tipo de envases. Las latas se cerraron y esterilizaron en autoclave a $115^{\circ} \mathrm{C}$ durante dos tiempos distintos: 55 minutos, tiempo suficiente desde el punto de vista microbiológico y 90 minutos, tiempo que suele utilizarse en la industria. Las latas se dejaron madurar 1 mes para que el líquido de cobertura se distribuyera uniformente y fuera absorbido por el contenido sólido, y entonces se procedió a la toma de muestra de las conservas $\left({ }^{*} S 55\right)$ y ( $\left.{ }^{*} S 90\right)$ Posteriormente, ambas conservas fueron almacenadas durante un año a temperatura ambiente, finalizado el cual se abrieron y analizaron, ( ${ }^{\star S 55 ~} 1$ año) y (S90 1 año*).

En la época en que correspondía realizar el estudio, el contenido de 12 envases de cada tipo de conserva se liofilizó, homogeneizó y se tomaron tres alícuotas de la mezcla para el análisis posterior.

\subsection{Análisis de las muestras.}

En esos triplicados de muestras de bonito crudo, cocido y en las distintas conservas se extrajo y cuantificó la grasa con éter de petróleo $40-60^{\circ}$ en una unidad extractora Soxtec System 1040 Tecator.

Para el análisis cromatográfico de los ácidos grasos procedentes de la grasa extraída del alimento, así como del aceite de cobertura de las conservas, se siguió la técnica de Metcalfe y col. (10) realizándose en tres etapas: saponificación con sosa en metanol $0,5 \mathrm{M}$, metilación de los ácidos grasos con $\mathrm{BF}_{3} / \mathrm{CH}_{3} \mathrm{OH}$ y extracción de los ésteres metílicos con hexano. Para la cuantificación de los ácidos grasos se utilizó un cromatógrafo de gases Hewlett-Packard 5720A. La fase estacionaria de la columna utilizada contenía supelcoport 2330 al $10 \%$ sobre chromosorb 100-120 (Supelco). La temperatura de la columna se mantuvo a $170^{\circ} \mathrm{C}$ durante 8 minutos, elevándose hasta $240^{\circ} \mathrm{C}$ a razón de $2^{\circ} \mathrm{C} /$ minuto. $\mathrm{El}$ detector e inyector se mantuvieron a $300^{\circ} \mathrm{C}$ y $250^{\circ} \mathrm{C}$ respectivamente. La cantidad de muestra inyectada fue $0.5 \mu \mathrm{l}$. Las áreas de los picos se calcularon con un integrador Perkin Elmer M-2 Minigrator 7123A. 
Los ácidos grasos fueron identificados comparando sus tiempos de retención absolutos y relativos con estándares comerciales (Sigma, St. Louis, USA), analizados bajo las mismas condiciones experimentales.

Para el tratamiento estadístico de los datos se realizaron análisis de varianza-covarianza (ANOVA). Las comparaciones entre pares de grupos se hicieron mediante el test " $t$ " modificado según el estadístico $F$. El nivel mínimo de significación se ha establecido en el $5 \%$ y se expresará como $\mathrm{P}<0.05$.

\section{3.-RESULTADOS Y DISCUSION}

El contenido graso del atún blanco (Tabla I) se aproximó al límite inferior del rango que las tablas de composición de alimentos señalan para túnidos, entre 0.96 y $15.5 \mathrm{~g} / 100 \mathrm{~g}$ de alimento; lo cual explica por qué la modalidad enlatada fue "lomos", en la que se elimina gran cantidad de carne roja, más rica en grasa.

Tras la cocción, y gracias a la pérdida de agua, se elevó ligeramente el porcentaje lipídico, en línea con lo que describen otros autores (11) (12).

Durante el proceso de esterilización se produjo una difusión de sustancias entre el alimento y el líquido de cobertura, como ya fue señalado por Petelina $y$ Artiukhona (1961) (13) y, al igual que en la fritura en baño de aceite, existió un intercambio entre el agua del pescado y la grasa del medio; de forma que ambos contenidos se hallaron inversamente correlacionados $(r=-0.9464)$ (14). Así la conserva de atún en aceite se fue enriqueciendo en grasa, convirtiéndose en un alimento más energético.

Pirazzoli y col. (1980) (15) hablan de que por el paso del tiempo en las conservas en aceite se incrementa la penetración del líquido circundante en el interior del tejido muscular. Por ello la fracción lipídica de las conservas de atún almacenadas se elevó, tanto más cuanto menor era su contenido graso tras la esterilización: es decir, el incremento fue mayor en la que sólo se había esterilizado durante 55 minutos, de forma que al cabo del año ambas ( 555 y S90 1 año) poseían el mismo porcentaje de grasa. Lo que se observa de forma más palpable cuando los resultados se expresan como porcentaje de la sustancia seca (Tabla I).

En el patrón de ácidos grasos de los lípidos del atún blanco crudo, que recuerda al de la grasa de otros pescados azules, predomina entre los saturados el palmítico, el oleico es el mayor componente de los monoinsaturados y entre los polinsaturados, los $\mathrm{n}-3: \mathrm{C} 20: 5, \mathrm{y}$, sobre todo, C22:6, suponen aproximadamente un $37 \%$ del total de ácidos grasos. Este hecho, unido al contenido medio bajo de $n-6$, hace que la

Tabla 1

Composición porcentual en grasa de muestras de bonito del norte crudo, cocido y en conservas recién preparadas y almacenadas

\begin{tabular}{|c|c|c|}
\hline & & \\
\hline & $\mathrm{g} / 100 \mathrm{~g}$ alimento & $\mathrm{g} / 100 \mathrm{~g} \mathrm{s.s}$. \\
\hline CRUDO & $1.90 \pm 0.25$ & $6.36 \pm 0.83$ \\
\hline COCIDO EN & $3.33 \pm 0.08$ & $9.24 \pm 0.21$ \\
\hline SALMUERA & & \\
\hline $\mathrm{S} 55$ & $6.34 \pm 0.15 * 0$ & $16.50 \pm 0.39 * 0$ \\
\hline S90 & $7.19 \pm 0.11 * 0 \Delta$ & $18.06 \pm 0.29 * 0 \triangle$ \\
\hline S55 & $9.79 \pm 0.36 * 0 \Delta$ & $23.30 \pm 0.85 * 0$ \\
\hline s9o & $9.59 \pm 0.32 * 0 \Delta$ & $23.00 \pm 0.77 * 0$ \\
\hline
\end{tabular}

Valores medios \pm desviación standard.

SS: Sustancia seca.

S55 y S90: Conservas esterilizadas 55 y 90 minutos respectivamente.

- Indica diferencias significativas $(P<0.05)$ respecto al bonito crudo.

0 Indica diferencias significativas $(P<0.05)$ respecto al bonito cocido en salmuera.

$\Delta$ Indica diferencias significativas $(\mathrm{P}<0.05)$ entre tiempos de esterilización a igualdad de las demás condiciones.

- Indica diferencias significativas $(P<0.05)$ entre tiempos de almacenamiento a igualdad de las demás condiciones. 
Tabla II

Modificaciones en la composición de ácidos grasos del bonito del norte tras distintas etapas de preparación y almacena-

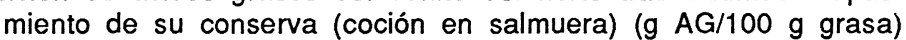

\begin{tabular}{|c|c|}
\hline ACIDO GHASO & CHUDO \\
\hline C14:0 & 3.1510 .3 \\
\hline C15:0 & 1.2510 .0 \\
\hline $\mathrm{C} 16: 0$ & $20.40 \pm 1.0$ \\
\hline C16:1 77 & 2.4010 .0 \\
\hline C17:0 & $1.70 \pm 0$. \\
\hline C18:0 & $6.60 \pm 0.3$ \\
\hline$C 18: 2=9$ & 15.1010 .70 \\
\hline $\mathrm{C} 18 ; 2=6$ & $1.50 \times 0.10$ \\
\hline$C 18: 3=3$ & $2.05 \pm 0.45$ \\
\hline$c 20: 1=9$ & $0.70 \pm 0.00$ \\
\hline$C 18: 4=3$ & $0.15 \pm 0.0$ \\
\hline$C 20: 4 w 6$ & $3.85 \pm 0.2$ \\
\hline$C 22: 2=6$ & $0.50 \pm 0.00$ \\
\hline$c 20: 5=3$ & $8.35 \pm 0.25$ \\
\hline C22:5 w3 & $1.90 \pm 0.10$ \\
\hline$C 22: 6 \approx 3$ & $28.70 \pm 1.80$ \\
\hline Total suturadoe & 33.1 \\
\hline Totel eunoinaturados & 18.2 \\
\hline Total politinaseturados & 47.0 \\
\hline PS/S & 1.98 \\
\hline$-3 / w 6$ & 7.03 \\
\hline
\end{tabular}

\begin{tabular}{|c|c|c|c|c|}
\hline $\begin{array}{c}\text { COCIDO EN } \\
\text { SALMUERA }\end{array}$ & 555 & $s 90$ & S55 1 Ano & S90 I Año \\
\hline 3.2010 .10 & $1.75 \pm 0.15 \neq 0$ & $\overline{1.60 \pm 0.14}=0$ & $1.50 \pm 0.0010$ & $1.0020 .10=00$ \\
\hline 0.9050 .10 & 0.2520 .05 & 0.2010 .00 & $0.60 \pm 0.00$ & $0.70 \pm 0.00$ \\
\hline $19.15 \pm 0.65$ & $16.3510 .15^{\star}$ & $15.75+0.05$ & $14.95+0.05 \pm 0$ & $13.6510 .75 * 0$ \\
\hline $3.40 \pm 1.00$ & $1.30 \pm 0.00$ & 1.1520 .05 & 1.2510 .45 & $0.55 \pm 0.15^{\circ}$ \\
\hline $1.25 \pm 0.35$ & $0.40 \pm 0.00$ & $0.20 \times 0.10^{\star}$ & $0.35 \pm 0.15^{\star}$ & $0.20 \pm 0.00$ \\
\hline $5.80 \pm 0.00$ & $5.00 \pm 0.00$ & $4.95 \pm 0.15$ & $4.35 \pm 0.35$ & $4.35 \times 0.35$ \\
\hline $17.40 \pm 0.30$ & $20.35 \pm 0.55$ & $20.3510 .45^{\star}$ & $21.70 \pm 0.80^{\star 0}$ & $20.95 \pm 0.15 * 0$ \\
\hline $1.20 \pm 0.20$ & $33.60 \times 1.60 \star 0$ & $32.00 \pm 0.10^{ \pm 0}$ & 35.2010 .0010 & $40.40 \pm 0.90 \$ 04$ \\
\hline $2.60 \pm 0.80$ & $1.50 \pm 0.00$ & $5.40 \pm 0.10^{10}$ & $5.70 \pm 0.20 \approx 0$ & $6.15 \pm 0.25 \neq 0$ \\
\hline $1.55 \times 0.35$ & $0.20 \pm 0.00$ & 0.7020 .30 & $0.40 \times 0.10$ & $0.30 \times 0.000$ \\
\hline $0.30 \pm 0.00$ & 0.2010 .00 & 0.2010 .00 & $0.10 \pm 0.00$ & $0.20 \pm 0.00$ \\
\hline $3.50 \pm 0.00$ & $1.20 \pm 0.10 \star 0$ & $1.20 \times 0.10^{* 0}$ & $1.05 \pm 0.15 * 0$ & $0.65 \pm 0.15 \bowtie 0$ \\
\hline $0.10 \pm 0.00$ & $0.10 \pm 0.00$ & 0.1510 .05 & $0.10 \pm 0.05$ & $0.10 \pm 0.00$ \\
\hline 0.0510 .15 & $3.4510 .15 * 0$ & $3.60 \pm 0.10 * 0$ & $2.65 * 0.0510$ & $2.30 \pm 0.10 \approx 0$ \\
\hline $2.15 \pm 0.25$ & 0.9510 .15 & $0.50 \pm 0.20 \star 0$ & $0.30 \pm 0.00$ & $0.15 \pm 0.05 \neq 0$ \\
\hline $29.05 \pm 1.15$ & $11.65 \pm 0.45^{ \pm 0}$ & $11.25 \pm 0.95 \pm 0$ & $8.65 \pm 0.05 \div 0$ & $7.40 \pm 0.20 \star 0$ \\
\hline 30.3 & 23.75 & 22.90 & 21.75 & 19.90 \\
\hline 22.3 & 21.85 & 22.20 & 23.35 & 21.80 \\
\hline 46.95 & 55.85 & 54.30 & 53.75 & 57.35 \\
\hline 2.29 & 3.27 & 3.34 & 3.54 & 3.98 \\
\hline 8.78 & 0.59 & 0.59 & 0.47 & 0.98 \\
\hline
\end{tabular}

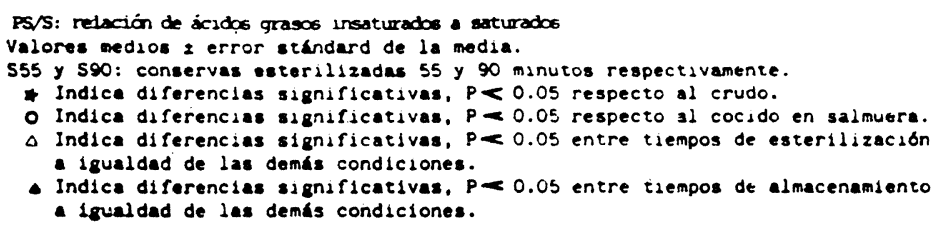

relación $n-3 / n-6$ se sitúe entre las de los pescados con cifras más elevadas, similar a la de la grasa del salmón y halibut, aunque inferior a la de la sardina (Tabla II).

En línea con la estabilidad descrita por otros autores en especies diferentes (11) (12), por efecto de la cocción la composición porcentual en ácidos grasos de los lípidos del atún blanco no mostró variaciones sustanciales; los $n-3$ y $n-6$, ofrecieron especial estabilidad $y$, por tanto, la relación $n-3 / n-6$ no se modificó apreciablemente (Tabla II). Como ya se ha dicho, a consecuencia del tratamiento se incrementó también el contenido lipídico en el alimento, de ahí que se elevaran asimismo cada uno de los ácidos grasos, más significativamente aquellos que son componentes mayoritarios de la fracción grasa del atún (Tabla III).

Tras la esterilización, coincidiendo con otros resultados (6) (16), la grasa de este pescado se empobreció porcentualmente en los ácidos propios y se enriqueció en los mayoritarios del aceite de soja utilizado como cobertura: este hecho fue fundamentalmente consecuencia de la dilución de la grasa del pescado en el aceite que penetra. Así, disminuyeron los saturados y los poliinsaturados de más de 20 átomos de $\mathrm{C}$, especialmente el C20:5 y, sobre todo, el C22:6. Paralelamente se incrementaron el oleico, linolénico $y$, de modo espectacular, el linoleico (Tabla II). En consecuencia, la grasa se hizo más insaturada a base del aumento de los PUFA preferentemente n$6 \mathrm{y}$, por ello, la relación PS/S creció, a la vez que disminuyó intensamente la relación $n-3 / n-6$. Por lo tanto, parece que la esterilización en aceite durante 55 ó 90 minutos, sin diferencias en razón del tiempo, introdujo cambios en la naturaleza de la grasa alimentaria al igual que se describe en otro proceso, la fritura en baño de aceite (7) (8).

En términos generales, los cambios en la composición del aceite de cobertura de las conservas (Tabla IV), obviamente, resultaron complementarios a los que se acaban de señalar en la grasa del alimento. En ellos se detectaron cantidades algo más importantes del ácido graso mayoritario en el atún blanco, C22:6 y pequeñas proporciones de los otros ácidos grasos típicos del pescado. En sentido inverso, sólo apareció una ligera disminución en el componente cuantitativamente más importante del aceite de soja, el ácido linoleico. 
Tabla III

Modificaciones en la composición de ácidos grasos del bonito del norte tras distintas etapas de preparación y almacenamiento de su conserva (coción en salmuera) (mg AG/100 g alimento)

\begin{tabular}{|c|c|c|c|c|c|c|}
\hline ACILU GHASO & CRUUO & $\begin{array}{r}\text { COCIDO EN } \\
\text { SALMUEFA }\end{array}$ & S55 & S90 & S55 \& año & S90 1 añu \\
\hline$C 14: 0$ & $57.9 \pm 6.4$ & $107.2 \pm 3.3^{\star}$ & $110.929 .5^{\star}$ & $130.3 \pm 7.2^{\star}$ & $146.8 \times 9.8^{\star}$ & 95.91 y.0 \\
\hline Clb:0 & $23.0 \pm 0.9$ & $30.1 \pm 3.3$ & $15.8 \pm 3.2$ & $14.4 \pm 7.2$ & $58.7 \pm 9.8$ & 67.119 .6 \\
\hline$c 16: 0$ & $375 \pm 18$ & $641 \pm 21 \star$ & $1036 \pm 9 \neq 0$ & $1140 \pm 3 \star 0$ & $1436 \pm 4$ \#० & $1309 \pm 71 \pitchfork 0$ \\
\hline C10:1 w7 & $44.1 \pm 1.8$ & $113.9 \pm 33.5$ & $82.4 \pm 6.3$ & $83.2 \pm 3.6$ & $122.3 \pm 44.0$ & 52.7114 .4 \\
\hline C17:0 & $31.2 \pm 7.4$ & $41.8 \pm 11.7$ & $25.3 \pm 6.3$ & $14.4 \pm 7.2$ & $34.2 \pm 14.7$ & $19.1 \pm 9.6$ \\
\hline C18:0 & $121.4 \pm 5.5$ & $194.3 \pm 3.3$ & $317.0 \pm 6.3$ & $358.3 \pm 10.9$ & $425.8 \pm 34.3$ & $417.1 \pm 33.6$ \\
\hline C18:1 w9 & $277 \pm 12$ & $582 \pm 10 \star$ & $1290 \pm 34 ٪ 0$ & $1473 \pm 32 \Vdash 0$ & $2124 \pm 78 \star 0 \Delta$ & $2009 \pm 14 \star 04$ \\
\hline Clu:2 wo & $27.6 \pm 1.8$ & 40.226 .7 & $2142 \pm 101 \star 0$ & $2316 \pm 7 \star 0$ & $3446 \pm 78 \bowtie 04$ & $3874186 \pitchfork 04$ \\
\hline$C 18: 3=3$ & $37.7 \pm 8.3$ & $87.1 \pm 26.8$ & $285.3 \pm 6.3^{\star 0}$ & $390.9 \pm 7.2^{\star 0 \Delta}$ & $558.0 \pm 19.6 * 0$ & $589.7 \pm 24.0 \star 0 \Delta$ \\
\hline cżi:l wy & $12.8 \pm 1.8$ & $51.9 \pm 11.7$ & $12.6 \pm 6.3$ & 50.6121 .7 & $39.1 \pm 9.8$ & $28.7 \pm 9.0$ \\
\hline C18:4 w3 & $2.7 \pm 0.9$ & $10.0 \pm 3.3$ & $12.6 \pm 6.3$ & $14.4 \pm 7.2$ & $9.7 \pm 9.8$ & $19.1 \pm 9.6$ \\
\hline C2u: 4 w6 & $70.8 \pm 4.6$ & $117.2 \pm 3.3^{\star}$ & $76.0 \pm 6.3$ & $86.8 \pm 7.2$ & $102.7+14.7$ & $62.3 \pm 14.4$ \\
\hline Cट22:2 w6 & $9.2 \pm 1.8$ & $3.3 \pm 3.3$ & $6.3 \pm 6.3$ & $10.8 \pm 3.6$ & $9.7 \pm 9.8$ & 9.519 .6 \\
\hline$c 20: 5 w 3$ & 153.614 .6 & $269.6 \pm 3.3^{\star}$ & $218.7 \pm 9.5$ & $260.6 \pm 7.2$ & $259.4 \pm 4.2$ & $220.5 \pm 9.6$ \\
\hline C22:5 w3 & $34.9 \pm 1.8$ & $72.0 \pm 8.4$ & $60.2 \pm 9.5$ & $36.2 \pm 14.5$ & $29.4 \pm 9.8$ & $14.3 \pm 4.8$ \\
\hline$C 22: 6 w 3$ & $528 \times 33$ & $973 \pm 38 \star$ & $738 \pm 28$ & $814 \pm 68$ & $846 \pm 4$ & $709+19$ \\
\hline
\end{tabular}

Valores medios z error standard de la media.

555 y 590 : conservas esterilizadas 55 y 90 minutos respectivamente.

Indice diferencias significativas, $P<0.05$ respecto al crudo.

Indica diferencles slenificativas, $P<0.05$ respecto al cocido en salmutra.

Indica diferencies significatives, $P<0.05$ entre tiempos de esterilización

- Iqualdad de las demás condiciones.

Indica diferencias significarivas, $p<0.05$ entre tiempos de almacenamiento

- lgualdad de las demás condiciones.

Tabla IV

Composición en ácidos grasos del aceite de soja utilizado y del aceite de cobertura de conservas de bonito del norte recién preparadas y tras un año de almacenamiento (g AG/100 g grasa)

\begin{tabular}{|c|c|c|c|c|c|}
\hline GRASO & ACEITE SOJA & AC. $\mathbf{S 5 5}$ & AC. 590 & AC. $\$ 551$ año & AC. S90 1 año \\
\hline $\mathrm{C} 14: 0$ & -- & $0.20 \pm 0.00$ & $0.30 \pm 0.00$ & $0.25 \pm 0.05$ & $0.15 \pm 0.05$ \\
\hline $\mathrm{C} 16: 0$ & $11.75 \pm 0.55$ & $11.60 \pm 0.10$ & $11.75 \pm 0.15$ & $13.70 \pm 2.90$ & $15.35 \pm 0.15$ \\
\hline $\mathrm{C} 18: 0$ & $3.70 \pm 0.14$ & $3.75 \pm 0.15$ & $4.00 \pm 0.00$ & $3.75 \pm 0.25$ & $3.30 \pm 0.00$ \\
\hline C18:1 w9 & $21.10 \pm 0.31$ & $20.50 \pm 0.20$ & $20.70 \pm 0.30$ & $21.10 \pm 0.90$ & $20.75 \pm 0.05$ \\
\hline $\mathrm{C} 18: 2$ w6 & $54.85 \pm 0.05$ & $52.00 \pm 0.00$ & $52.50 \pm 0.20$ & $49.65 \pm 0.65$ & $52.50 \pm 0.30$ \\
\hline C18:3 w3 & $7.20 \pm 0.40$ & $7.00 \pm 0.30$ & $7.30 \pm 0.20$ & $6.70 \pm 0.25$ & $7.00 \pm 0.20$ \\
\hline C20:1 w9 & -- & $0.50 \pm 0.10$ & $0.20 \pm 0.00$ & --- & -- \\
\hline $\mathrm{C} 20: 4$ w6 & -- & $0.20 \pm 0.10$ & $0.20 \pm 0.10$ & $0.15 \pm 0.15$ & -- \\
\hline $\mathrm{C} 20: 5$ w3 & --- & $0.35 \pm 0.15$ & $0.45 \pm 0.05$ & $0.15 \pm 0.15$ & -- \\
\hline C22:6 w3 & --- & $1.30 \pm 0.20$ & $0.90 \pm 0.10$ & $0.90 \pm 0.50$ & $0.20 \pm 0.20$ \\
\hline
\end{tabular}

Valores medios \pm error stándard de la media.

AC.: Aceite de cobertura de la muestra que se indica.

S55 y S90: Conservas esterilizadas 55 y 90 minutos respectivamente. 
A pesar de estas modificaciones en la composición porcentual de la grasa, teniendo en cuenta su magnitud en valor absoluto $y$ el enriquecimiento lipidico de la conserva, en el atún enlatado que es lo que se ingiere, respecto del pescado cocido, se observaron los siguientes cambios:

- Los ácidos grasos saturados no se alteraron claramente, salvo el C16:0, componente abundante también en el aceite de soja, que se incrementó. Algo parecido ocurrió con los monoinsaturados, el C16:1 y el C20:1 que tendieron a disminuir, mientras que el oleico se elevó significativamente.

- Sin duda, fue en los poliinsaturados donde la difusión entre ácidos grasos del pescado y del medio se hizo más palpable. Existió una leve tendencia a la disminución en los ácidos grasos de cadena larga del atún, preferentemente en el C20:5 y sobre todo, en el C22:6, aunque este último se retuvo aproximadamente en un $75-80 \%$ en ambas modalidades de conservas ensayadas (Figura 1). A la vez, y como consecuencia de la entrada de aceite de cobertura, la conserva aumentó extraordinariamente su contenido en linoleico, y triplicó en S55 o cuadriplicó en S90 el de linolénico, siendo ésta la diferencia más acusada entre ambas conservas por efecto del tiempo de esterilización (Figura 2).

Como resultado global de todo el proceso, el atún blanco en ambas conservas contenía mayor cantidad

\section{Figura 1}

Modificaciones del contenido de eicosapentaenoico y docosahexaenoico durante la preparación y almacenamiento de la conserva de atún blanco ( $\mathrm{mg} / 100 \mathrm{~g}$ alimento).

*Indica diferencias significativas, $\mathrm{P}<0.05$ respecto al $\mathrm{BN}$ crudo.

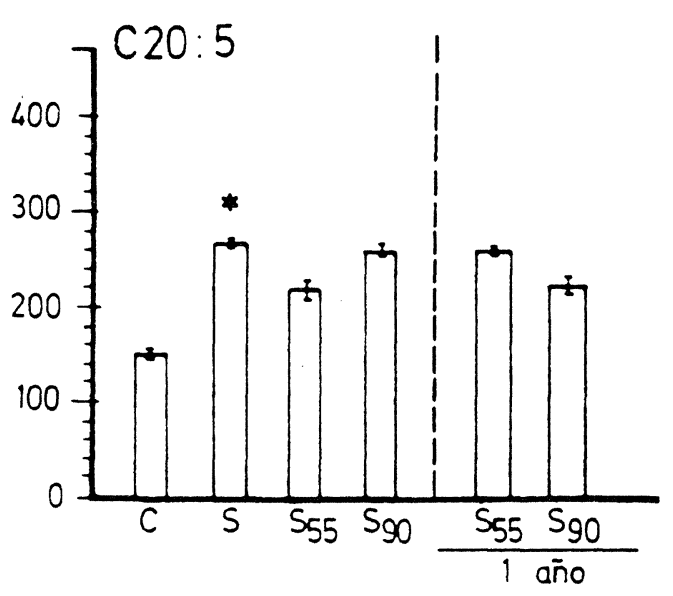

de cada uno de los ácidos grasos que la muestra cruda o cocida, exceptuando algunos poliinsaturados; y lo más destacable, había sufrido un gran enriquecimiento en los insaturados mayoritarios del aceite de soja.

Estos intercambios que ocurrieron durante la esterilización se agudizaron tras el año de almacenamiento y así el análisis de la grasa reveló una tendencia a la disminución de los poliinsaturados propios $y$ al incremento de los del aceite de soja, especialmente del C18:2 (Tabla II), que en el alimento, más enriquecido en grasa, se visualizó mejor y se tradujo en un aumento significativo en ambas modalidades de los contenidos no sólo del linolénico sino también del linoleico e incluso del oleico (Tabla III). Parece claro que en el atún blanco en conserva, más aún si se almacena durante un año, sigue predominando la grasa insaturada, pero ahora ya con una relación $n$ $3 / n-6$ muy disminuida.

A este respecto conviene recordar que la importancia de los pescados grasos en las enfermedades

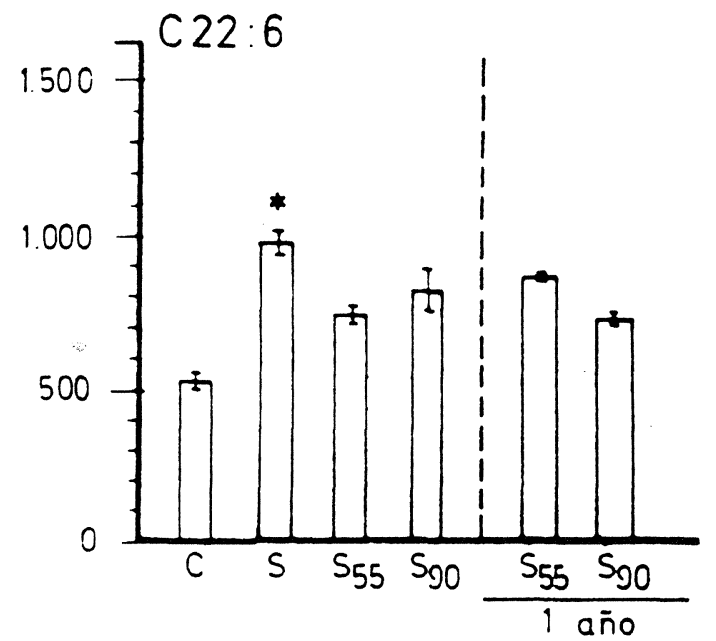

cardiovasculares se relaciona con estos índices y así Huang y col. (1986) (17) consideran que la reducción de los lípidos sanguíneos de animales sólo se produce cuando sus dietas se suplementan con grasas de pescado de relaciones $n-3 / n-6$ no inferiores a 0,39 . Por otra parte, estudios epidemiológicos y metabólicos recientes (18) han demostrado que los ácidos grasos monoinsaturados tienen un papel importante en la prevención de enfermedades cardiovasculares pues parece que ejercen ciertos efectos beneficiosos de los PUFA pero sin algunos de sus inconvenientes. Por lo que actualmente se piensa que el reparto de saturados, monoinsaturados y poliinsaturados más idóneo para la salud sería el de 1/1/1 (19), proporciones lejanas a la que presentan las conservas de atún blanco enlatado en aceite de soja.

A la vista de estas consideraciones sería importante elegir convenientemente el aceite de cobertura y para el atún blanco posiblemente el de oliva resultaría más idóneo, porque al penetrar preferentemente el ácido oleico (6) de la familia $n-9$, se conseguiría 

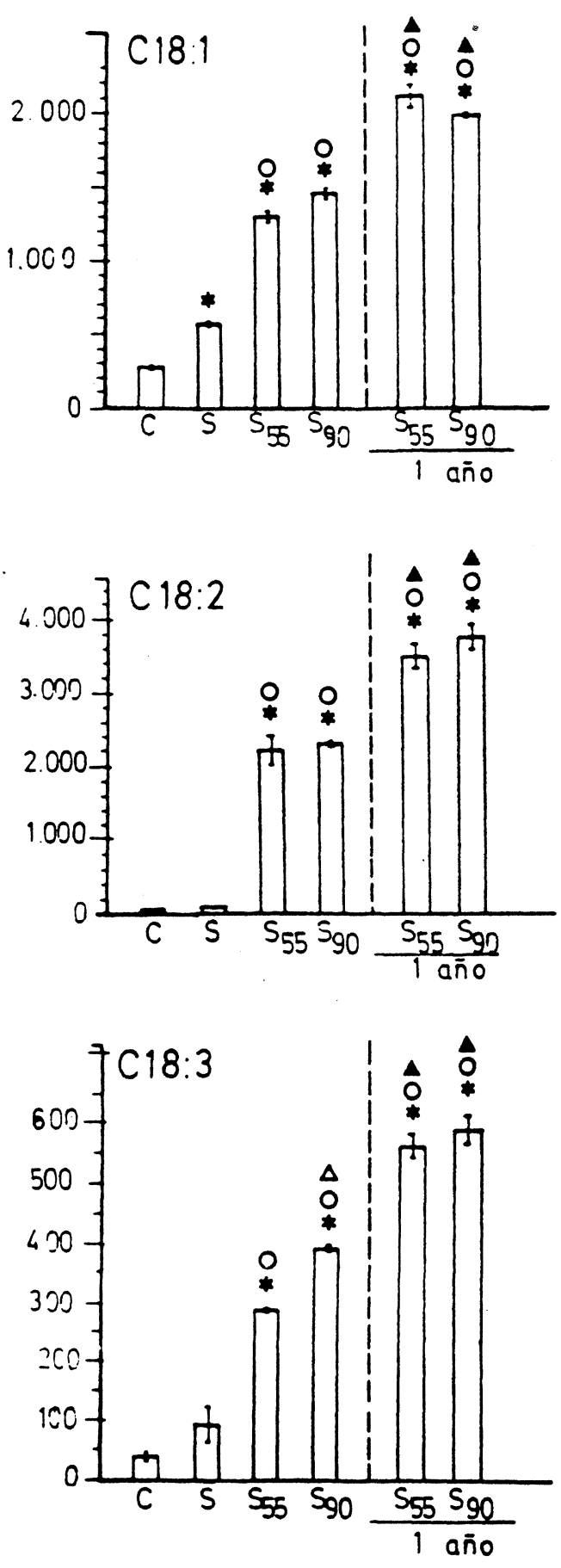

Figura 2

Modificaciones en la composición de algunos ácidos grasos del atún blanco tras distintas etapas de preparación y almacenamiento de su conserva (mg/100 $\mathrm{g}$ alimento)

- Indica diferencias significativas, $P<0.05$ respecto al $B N$ crudo.

- Indica diferencias significativas $\mathrm{P}<0.05$ respecto a su modalidad de cocción.

$\Delta$ Indica diferencias significativas, $P<0.05$ entre tiempos de esterilización a igualdad de las demás condiciones.

- Indica diferencias significativas, $P<0.05$ entre tiempos de almacenamiento a igualdad de las demás condiciones. un alimento con una relación $n-3 / n-6$ menos alterada y con un contenido mayor de monoinsaturados, con lo que se mantendría la relación ácidos grasos saturados/monoinsaturados/poliinsaturados a un nivel más conveniente. Las conservas así preparadas serían sin duda un alimento especialmente equilibrado para incluirlo en dietas cardiosaludables según los criterios establecidos en el II Congreso Mundial Vasco sobre "Problemas de la nutrición en las sociedades desarrolladas".

\section{AGRADECIMIENTOS}

Este trabajo ha sido financiado por los proyectos N. 516 de la CAICYT y N.- ALI88-0255 de la CICYT. Gracias al Dr. Sánchez-Muniz por su ayuda en la interpretación de los resultados, al Instituto de Investigaciones Marinas donde se enlató la conserva y a D. a Isabel Orvay y D. ${ }^{\text {a }}$ Elena Asensio por su inestimable ayuda en la confección del original.

\section{BIBLIOGRAFIA}

1. Von Lossonczy, T.O.; Ruiter, R. A.; Bronsgeest-Schoutz, H. C Van Gent, C. M., and Hermus, R. J. J.-"The effect of a fish diet on serum lipids in healthy human subjects".- Am J. Clin. Nutr. 31 (1978) 1340

2. Harris, W. S.; Connor, W. E., and McMurry M. P.- "The comparative reduction of the plasma lipids and lipoproteins by dietary polyunsaturated fats: salmon oils versus vegetable oils".Metabolism. 32 (1983) 179-184.

3. Varela, G.; Moreiras-Varela, O., y Requejo, A.- "Estudios de Nutrición".- Vol. I y II. Publicaciones del Instituto Nacional de Estadistica. Madrid, 1985.

4. Younathan, M. T.; Marjan, Z. M., and Arshad, F. B.- "Oxidative rancidity in stored ground turkey and beef".- J. Food Sci. 45 (1980) 274-276.

5. Younathan, M. T.; Oon, J. K., and Yusof, R. B. M.- "Control of heat induced oxidative rancidity in refrigerated Shark and Mackerel".- J. Food Sci. 48 (1983) 176-178.

6. Garcia Miranda, M. P.- "Contribución al estudio de sardinas de mercado español y del aceite de cobertura de sus conservas. III) Composición en ácidos grasos de la sardina en conserva y sus aceites de cobertura".- Tesina de Licenciatura. Facultad de Farmacia. Universidad Complutense de Madrid, 1984.

7. Varela, G.- "Influence of household handling".- Bibliotheca Nutr Dieta 34 (1985) 9-25

8. Sánchez-Muniz, F. J.; Hernández, I., y Cuesta, C.- "Estudio de la calidad del aceite extraido de patatas fritas en aceite de oliva".- Grasas y Aceites 40 (1989) 399-405.

9. Mai, J.; Shimp, J.; Weihrahch, J., and Kinsella, J. E.- "Lipids of fish fillets: changes following cooking by different methods" J. Food Sci. 43 (1978) 1669

10. Metcalfe, L. V.; Schmitz, A. A.; Pelka, J. R.- "Rapid preparation of fatty acid esters from lipids for gas chromatographic analysis".- Anal. Chem. 38 (1966) 514-515.

11. Gall, K. L.; Otwell, W. S.; Koburger, M., and Appledorf, H."Effects of four cooking methods on the proximate mineral and fatty acid composition of fish fillets".- J. Food Sci. 48 (1983) 1068-1074.

12. Beamonte, A.- "Estabilidad del triptófano durante los procesos térmicos culinarios de preparación de un pescado graso (Sar- 
dina pilchardus). Influencia del almacenamiento al estado congelado".- Tesina de Licenciatura. Universidad Complutense de Madrid, 1989.

13. Petelina, A. L., and Artiukhoua, S. A.- "Condiciones óptimas para fabricar conservas de sardina del Atlántico en aceite".FAO, Extractos de la Pesca Mundial 7 (1961) 37.

14. García Arias, M. T.- "Influencia del modo de cocción y tiempo de esterilización y almacenamiento en el valor nutritivo de conservas de atún blanco".- Tesis Doctoral. Universidad Complutense de Madrid, 1989.

15. Pirazzoli, P., Abroggi, F. and Incerti, I.- "Conserve di tonno all'olio: variazione della composizione in funzione del tipo di cottura e infuenza della temperatura di magazzinaggio sulla maturazione".- Ind. Conserve 55 (1980) 279-285.

16. Yamamoto, Y. and Imose, K.- "Changes in fatty acid composition in sardines (sardinops melanosticta) with cooking and refrigerated storage".-J. Nutr. Sci. Vitaminol 35 (1989) 39-47.

17. Huang, Y. S.; Nassar, B. A., and Horrobin, D. F.- "Changes of plasma lipids and long chain n-3 and n-6 fatty acids in plasma, liver, heart and kidney phospholipids of rats fed variable level of fish oil with or without cholesterol supplementation".- Biochem. Biophys. Acta 879 (1986) 22-27.

18. Grundy, S. M.- "Monounsaturated fatty acids, plasma cholesterol and coronary heart disease".- Am. J. Clin.Nutr. 45 (1987) 1168.

19. Simopoulos, A. P.- "Executive summary, conference high-lights conclusions and recommendations".- Am. J. Clin. Nutr. 45 (1987) 1029.

(Recibido: Julio 1990) 